\title{
AVALIAÇÃO DE AMBIENTES VIRTUAIS DE APRENDIZAGEM: UMA REFLEX ÃO SOBRE O MODELO INTERACIONISTA E CONSTRUTIVISTA
}

Fernanda Borges Vaz Ribeiro, PPGAU/UFSC, fernandavaz23@ hotmail.com Marilda Todescat, CAD/UFSC, marildat@gmail.com

Alessandra de Linhares Jacobsen, CAD/UFSC, alessandradelinharesjacobsen@gmail.com

\section{Resumo}

A avaliação de ambientes virtuais de aprendizagem (AVAS) é realizada por meio de metodologias capazes de analisar a concepção de um AVA, de maneira a assegurar a sua qualidade e eficácia. Nesse sentido, este artigo faz uma análise reflexiva sobre o modelo interacionista/construtivista proposto por Schlemmer e Fagundes (2000) e apresentado na íntegra por Schlemmer (2002), que propõe o uso de parâmetros para avaliação de AVAS, sob o ponto de vista técnico, administrativo, comunicacional-social e didáticopedagógico. A metodologia utilizada foi a pesquisa bibliográfica. Contudo, a análise permitiu verificar que para realizar uma avaliação é essencial conhecer os princípios educacionais que norteiam o design instrucional do sistema, analisar suas múltiplas ferramentas e funcionalidades, e identificar como os usuários estão fazendo uso dos recursos do AVA, no intuito de verificar suas necessidades e ampliá-los.

Palavras-chave: Ambientes Virtuais de Aprendizagem. Avaliação. Modelo Interacionista e Construtivista.

\section{EVALUATION OF VIRTUAL LEARNING ENVIRONMENTS: A REFLECTION ON THE INTERACTIONAL AND CONSTRUCTIVIST MODEL}

\begin{abstract}
The evaluation of virtual learning environments (VLE) is performed by methodologies capable of examine the conception of a VLE, so as to ensure their quality and efficacy. Therefore, this article is a reflective analysis of the interactional and constructivist model proposed by Schlemmer and Fagundes (2000) and presented in full by Schlemmer (2002), which proposes the use of parameters for evaluation of VLE, from a technical, administrative, social-communication and pedagogical and didactic points of view. The methodology used was bibliographical search. However, the analysis has shown that to make a evaluation is essential to know the educational principles that guide the instructional design of the system, analyze your multiple tools and features, and identify how users are making use of the VLE resources, in order to verify their needs and expand them.
\end{abstract}

Keywords: Virtual Learning Environments. Evaluation. Interactional and Constructivist Model.

\section{INTRODUÇÃO}

A educação vivencia um momento em que a combinação de pequenos ajustes nas formas culturais de aprender e ensinar não é mais suficiente para enfrentar os desafios das novas gerações, que se encontram imersas em uma nova realidade tecnológica. Sendo assim, é necessário fazer uma mudança profunda nas estruturas e hábitos dos antigos métodos educacionais (Cóll; Monereo, 2010). 
Neste contexto, como um novo espaço oportunizado pelas tecnologias da informação e da comunicação, surgem os AVAS, isto é, um ambiente educacional informatizado de aprendizagem em rede, estruturado como sistemas de ensino e aprendizagem integrados, capazes de promover a participação do aluno neste processo educacional (kenski, 2014; Peters, 2012; Guimarães; Dias, 2014).

Para Haguenauer, Lima e Cordeiro Filho (2010), o AVA é uma ferramenta com uma perspectiva pedagógica, na qual o docente tem a possibilidade de disponibilizar diversos recursos para o aluno, bem como, textos, aulas, cronogramas e exercícios, entre outros. Nesse ambiente, o docente deve inserir a dinâmica das relações sociais, por meio de fóruns temáticos, chats e grupos de discussão. A comunicação pode ser fomentada de modo síncrono, ou seja, em tempo real, ou assíncrono quando alunos, professores e colegas podem efetuar a comunicação em tempos distintos, como blogs ou mural virtual.

Nesse contexto, é grande e crescente a quantidade e diversidade de AVAS disponíveis em diversas Instituições/Organizações como instrumentos facilitadores do processo de ensino-aprendizagem, com o propósito de transmitir a informação, propiciar a construção e o compartilhamento do conhecimento (Tori, 2010; Piva Jr. et al., 2011).

Deveras, como consequência desse crescimento exponencial de AVAS é necessário que as instituições e organizações que se propõem a utilizar esses sistemas, criem metodologias avaliativas por meio do estudo e análise das ferramentas e recursos disponibilizados; além dos benefícios, das facilidades e das oportunidades propiciadas aos usuários desses ambientes; outrossim, de suas especificações técnicas e do custo. (Schlemmer; Saccol; Garrido, 2015a, 2015b).

Todavia, quando uma instituição ou organização opta por escolher um AVA, é necessário um estudo prévio, realizado por uma equipe multidisciplinar que compõe o planejamento e estruturação das atividades de um curso ou programa, levando em consideração todas as particularidades, no que tange a parte técnica do software (ferramentas e funcionalidades), os documentos e orientações didático-metodológicas da instituição da qual fazem parte, no intuito de produzir e/ou organizar determinado conteúdo.

Entretanto, quando o AVA já foi estruturado e implantado em uma Instituição, é imprescindível a sua avaliação constante, com o propósito de verificar os pontos positivos e negativos apresentados pelo software, por meio da interação dos seus participantes, da aprendizagem, da colaboração e compartilhamento de informações, atividades e conhecimentos e, essencialmente, quando a avaliação abrange a utilização do software pelos discentes (Schlemmer; Saccol; Garrido, 2015a, 2015b; Oliveira; Costa; Moreira, 2001).

A princípio, para o desenvolvimento deste artigo, estudou-se trabalhos relacionados à avaliação de AVAS, baseados em Schlemmer (2001,2002, 2005), Schlemmer e Fagundes (2000), Schlemmer Saccol e Garrido (2015) e Trein e Schlemmer (2015). A posteriori, analisou-se o modelo interacionista/construtivista proposto, inicialmente, por Schlemmer e Fagundes (2000) e apresentado na íntegra por Schlemmer (2002), que propõe o uso de parâmetros que possibilitam avaliar AVAS, sob o ponto de vista técnico, administrativo, comunicacional-social e didático-pedagógico.

\section{AVALIAÇÃO DE AVAS}

A avaliação de AVAS estabelece metodologias avaliativas por meio do estudo e análise das ferramentas e recursos disponibilizados, dos benefícios, das facilidades e das oportunidades propiciadas aos participantes desses ambientes, além de suas especificações técnicas e do custo. Desse modo, é fundamental elaborar técnicas capazes de avaliar a concepção de AVAS, de maneira a assegurar a qualidade e a eficácia proposta pelos ambientes (Schlemmer; Saccol; Garrido, 2015a, 2015b). 
De acordo com Oliveira (2001), os instrumentos de avaliação de AVAS são essenciais e dão um feedback aos desenvolvedores e educadores sobre a multiplicidade de aspectos presentes no ambiente, bem como, sua usabilidade, ergonomia, confiabilidade, acessibilidade, interação e do ponto de vista pedagógico.

Em outras palavras, a interface destes softwares deve ser amigável e intuitiva de modo a facilitar e otimizar o seu uso e reduzir o processo exaustivo de busca de acesso a informação pelo usuário. Sendo assim, a avaliação é uma tarefa árdua e complexa, pelo fato dos AVAS estarem em constante evolução, apresentando variantes tecnológicas e de aprendizagem (Galvão et al., 2013; Oliveira, 2001).

Portanto, no âmbito metodológico, um grande desafio para a avaliação de AVAS está inserido no desenvolvimento de procedimentos de avaliação, que possibilitam estabelecer análises e julgamentos sobre os parâmetros pedagógicos inerentes a eles, de maneira a assegurar a adequação e a estabilidade dos AVAS no processo pedagógico (Galvão et al., 2013).

Sendo assim, as metodologias qualitativas direcionadas para a avaliação dos AVAS tem se evidenciado pelo emprego de estruturas avaliativas voltadas à identificação de uma multiplicidade de informações que oferecem contribuições ao processo de análise educacional do AVA (Galvão et al., 2013).

Em relação aos modelos de avaliação pode-se dizer que são considerados um guia que orienta a prática da avaliação. É um conjunto de concepções sobre os conceitos e a estrutura do trabalho de avaliação que disponibilizam indicadores para alcançar descrições, julgamentos e recomendações sustentáveis (Madaus; Kellaghan, 2000 apud Ruhe; Zumbo, 2013).

$\mathrm{Na}$ atualidade, muitas avaliações de AVAS vem sendo realizadas, entretanto, a grande maioria fazem uso de critérios baseados na quantidade de características técnicas que os sistemas possuem; ao passo que outras apresentam determinadas preocupações com o aspecto didático-pedagógico do software,

\begin{abstract}
pois todo e qualquer desenvolvimento de um produto para educação é permeado por uma concepção epistemológica, ou seja, por uma crença de como se dá a aquisição do conhecimento, de como o sujeito aprende. Essa concepção é a base do desenvolvimento do processo educacional, que é expresso nas ações educativas (Schlemmer; Saccol; Garrido, 2015b, p. 79).
\end{abstract}

Atualmente, duas concepções didático-pedagógicas consideradas fundamentais, porém, distintas, podem ser identificadas nos AVAS disponíveis nas Instituições de Ensino (IEs): a empirista e a interacionista/construtivista. Essas duas concepções apresentam posições completamente distintas, no que tange ao modo que o conhecimento é adquirido pelo sujeito, isto é, sobre como se dá a aprendizagem. Em resumo, pode-se distinguir uma da outra por suas potencialidades de interatividade: a concepção interacionista/construtivista fomenta uma interatividade comunicacional, ou seja, com uma linguagem simétrica, possibilitando ao usuário fluência e colaboração; contudo, no modelo empirista, a condição de interatividade é restrita ao simples ato de interação, entendida como a relação que proporciona ao usuário unicamente operar o sistema (Britain; Liber, 1999).

$\mathrm{Na}$ próxima subseção, apresentar-se-á o modelo de avaliação interacionista/construtivista de AVAS.

\title{
2.1 MODELO DE AVALIAÇÃO INTERACIONISTA/CONSTRUTIVISTA DE AVAS
}

Atualmente, diversas avaliações comparativas de AVAS vem sendo realizadas, contudo, a grande parte delas fazem uso de critérios limitados, tendo como base o número de características técnicas relevantes apresentadas pelo sistema; embora, outros modelos 
de avaliação apresentem preocupação com o aspecto didático-pedagógico e $\mathrm{o}$ administrativo (Schlemmer; Fagundes, 2000).

Desse modo, uma visão mais abrangente e sistêmica deve ser utilizada para a análise dos AVAS, em especial, a concepção epistemológica sobre a qual os mesmos foram desenvolvidos, a partir da funcionalidade, usabilidade e avaliação do sistema, sobretudo, quanto ao contexto humano ou dos sistemas organizacionais que apresentam o aspecto humanizador como uma de suas características essenciais (Schlemmer; Fagundes, 2000).

Em verdade, um olhar mais holístico sobre a avaliação se faz necessário para que se identifique os princípios educacionais que foram utilizados para construir o design instrucional dos AVAS, e além disso, como os usuários desses ambientes (educadores e aprendizes) podem fazer uso de seus recursos e ampliá-los (Schlemmer; Fagundes, 2000)

Nesse sentido, o modelo epistemologicamente interacionista/construtivista, proposto por Schlemmer e Fagundes (2000) e apresentado na íntegra por Schlemmer (2002), tem como estratégia avaliar por meio das perspectivas técnica, didáticopedagógica, comunicacional/social e administrativa, levando em consideração três ações fundamentais:

a) fomentar a melhoria da qualidade da aprendizagem, utilizando-se de métodos não convencionais;

b) dar suporte à processos comunicacionais que possibilitem um alto nível de interatividade, fortalecendo o trabalho em equipe;

c) reduzir a sobrecarga administrativa dos professores, possibilitando que façam o gerenciamento mais ágil de sua carga de trabalho, permitindo-lhes maior dedicação às necessidades educacionais individuais dos discentes.

O modelo de avaliação de AVAS interacionista/construtivista entende que:

o conhecimento é como uma relação de interdependência entre o sujeito e seu meio. Tem um sentido de organização, estruturação e explicação a partir do experenciado. É construído a partir da ação do sujeito sobre o objeto de conhecimento, interagindo com ele, sendo as trocas sociais condições necessárias para o desenvolvimento do pensamento (Schlemmer, 2001, p. 11).

Deveras, a partir da perspectiva interacionista/construtivista, o objeto de estudo do conhecimento engloba tudo sobre o sujeito, isto é, o meio físico, o meio simbólico e o meio social. Sendo assim, um sujeito realiza diversas trocas com seu meio, e assim, ambos formam juntos um sistema. O meio, ou o objeto existem, independentes do sujeito, contudo, não podem ser concebidos, senão, por aproximações, por meio da atividade física ou simbólica. Estas aproximações podem gerar alterações na estrutura cognitiva do sujeito tanto em nível de pensamento quanto em nível de ação (Chiarottino, 1988).

Portanto, a aprendizagem não ocorre simplesmente pela transmissão de algo que está fora, contudo, depende do processo de interação, da ação do sujeito sobre o objeto do conhecimento, dos esquemas de significação que permitem estabelecer relações com o novo (Schlemmer, 2001).

Contudo, pode-se dizer que o processo de ensino-aprendizagem em AVAS está centrado na interação, capaz de fomentar a aprendizagem, na construção do conhecimento, que ocorre num processo de ação/interação entre sujeito e objeto. $\mathrm{O}$ professor não é mais considerado o foco na ordem dos conteúdos, mas, está presente nos questionamentos e problematizações oriundos dos próprios sujeitos da aprendizagem, impulsionando a pesquisa e a busca de solução de problemas, relacionando-os com as suas experiências de vida (Trein, Schlemmer, 2009). 
Diante dessa contextualização sobre a concepção interacionista/construtivista apresentar-se-á as perspectivas técnica, comunicacional-social, administrativa e didáticopedagógica para avaliação de um AVA.

\subsubsection{Perspectiva técnica}

A perspectiva técnica analisa o suporte tecnológico que o sistema deve apresentar, incluindo ferramentas que permitem o gerenciamento do ambiente, bem como, suporte tecnológico para as ferramentas disponibilizadas pelo AVA, bem como, as ferramentas de autoria, de trabalho individual e coletivo e serviços diversos.

De acordo com Schlemmer (2005), o ambiente deve agregar espaços, possibilitando a construção do saber, a livre exploração, a descoberta, atuando como um ponto de encontro para os usuários se reunirem e desenvolverem atividades colaborativas que suscitem a construção do conhecimento.

Em sua análise, Schlemmer (2005) aponta ferramentas que devem constar em um AVA: ferramentas de gerenciamento e gestão de ambiente, ferramentas de gestão de comunidades, ferramentas de autoria (professor e/ou estudante), ferramentas da área individual (webfólio individual), ferramentas da área da comunidade (webfólio coletivo), ferramentas de serviços, ferramentas de interação síncrona, ferramentas de interação assíncrona e ferramentas de avaliação (Schlemmer, 2005).

\subsubsection{Perspectiva comunicacional-social}

Conforme a abordagem construtivista-social, é essencial que uma das maiores preocupações seja com a comunicação, pois é necessário que a interação aconteça entre os usuários envolvidos no processo de aprendizagem, possibilitando a interação e a construção colaborativa de possibilidades, dentro do ambiente, conduzindo à construção do conhecimento de maneira cooperativa. De acordo com essa constatação, o quadro 1 apresenta os itens que devem estar presentes em um AVA quanto ao aspecto comunicacional-social (Schlemmer, 2005).

Quadro 1- Perspectiva Comunicacional-social

\begin{tabular}{|c|c|}
\hline Item & Descrição \\
\hline Espaço informal & Permite obter informações gerais do ambiente e das comunidades. \\
\hline Fontes de negociação & $\begin{array}{l}\text { Possibilita a realização de um planejamento participativo e flexível. Os estudantes } \\
\text { podem negociar seus "contratos de aprendizagem". }\end{array}$ \\
\hline $\begin{array}{l}\text { Ferramentas } \\
\text { discursivas }\end{array}$ & $\begin{array}{l}\text { As ferramentas de comunicação são integradas. É possível dentro do mesmo } \\
\text { ambiente abrir um material disponibilizado e realizar um processo de conversação } \\
\text { sobre o assunto. Ambas as concepções (do professor e dos alunos) põem ser } \\
\text { acessadas por todos. }\end{array}$ \\
\hline Interação & $\begin{array}{l}\text { Interação mútua. As ações interdependentes influenciam o comportamento do } \\
\text { outro e tem seu comportamento influenciado a cada evento comunicativo, } \\
\text { possibilitando que a relação se transforme. }\end{array}$ \\
\hline Interatividade & $\begin{array}{l}\text { Permite o fornecimento de feedback significativo para ações de acordo com os } \\
\text { objetivos. Além disso, possibilita que os estudantes reestruturem o material. }\end{array}$ \\
\hline Aluno & $\begin{array}{l}\text { É visto como produtor ativo, agente do processo de aprendizagem e sujeito com } \\
\text { conhecimentos prévios. }\end{array}$ \\
\hline Professor & $\begin{array}{l}\text { É visto como mediador, coparticipante, explorador, investigador, facilitador, } \\
\text { instigador, problematizador. }\end{array}$ \\
\hline
\end{tabular}

Fonte: Adaptado de Schlemmer, 2005. 


\subsubsection{Perspectiva administrativa}

Esta perspectiva considera questões condizentes à administração dos usuários dentro do AVA e ao papel dos diferentes atores. Tem como seu cerne as seguintes perspectivas administrativas:

a) Adaptabilidade - possibilita ao professor adaptar as atividades de acordo com as necessidades individuais ou em grupos.

b) Monitoramento - possibilita que o professor monitore se a aprendizagem está ocorrendo e de que maneira está ocorrendo.

c) Organização - permite a criação de grupos para a organização dos alunos sem requerer alguma ação do professor.

d) Estrutura - em rede, interdisciplinar. A organização se dá por áreas/células/disciplinas temas.

\subsubsection{Perspectiva didático-pedagógica}

A perspectiva didático-pedagógica analisa as questões epistemológicas e os paradigmas educacionais que fundamentam a criação de um AVA. Entretanto, quando o contexto do ambiente é inerente ao processo educativo, o item mais relevante a ser avaliado é o critério didático-pedagógico do software, tendo em vista que qualquer desenvolvimento de um produto para educação é delineado pela concepção de aquisição do conhecimento, isto é, de que maneira o sujeito internaliza a aprendizagem.

A partir da análise de Schlemmer (2005), no quadro 2, apresentar-se-á os itens relevantes e as características de acordo com a perspectiva didático-pedagógica, considerando o paradigma Interacionista /construtivista.

\section{Quadro 2 - Perspectiva didático-pedagógica}

\begin{tabular}{|l|l|}
\hline \multicolumn{1}{|c|}{ Item } & \multicolumn{1}{|c|}{ Descrição } \\
\hline Foco no sistema & $\begin{array}{l}\text { Foco na aprendizagem, na construção do conhecimento na } \\
\text { colaboração, na cooperação, na autonomia, no desenvolvimento de } \\
\text { competências e habilidades, no respeito ao ritmo de desenvolvimento } \\
\text { de cada usuário. }\end{array}$ \\
\hline Visão sobre o aluno & $\begin{array}{l}\text { O aluno é visto como co-autor da comunidade, agente do processo de } \\
\text { aprendizagem, sujeito com conhecimentos prévios, pesquisador, } \\
\text { autônomo, participativo, cooperativo e crítico. }\end{array}$ \\
\hline Visão sobre o professor & $\begin{array}{l}\text { O professor é visto como mediador, coparticipante, explorador, } \\
\text { investigador, facilitador, instigador, problematizador, orientador, } \\
\text { articulador do processo de aprendizagem. Apresenta comportamento } \\
\text { interativo, é um educador, animador da inteligência, da aprendizagem }\end{array}$ \\
\hline $\begin{array}{l}\text { Ambiente de } \\
\text { aprendizagem }\end{array}$ & $\begin{array}{l}\text { Deve ser flexível, sem um controle centralizado vertical, participativo, } \\
\text { centrado na interação, na relação. Ponto de encontro para trocas, } \\
\text { construção do conhecimento e trabalho cooperativo. Favorece a } \\
\text { autonomia e o trabalho interdisciplinar. }\end{array}$ \\
\hline $\begin{array}{l}\text { Aquisição de } \\
\text { conhecimento }\end{array}$ & $\begin{array}{l}\text { O sistema permite o desenvolvimento de práticas pedagógicas } \\
\text { interacionistas, problematizadoras, centradas na pesquisa e } \\
\text { manipulação, no aprender e pensar. Auxilia na identificação e } \\
\text { resolução de problemas, proporcionando o trabalho cooperativo. }\end{array}$ \\
\hline Avaliação & $\begin{array}{l}\text { O sistema permite uma avaliação com foco no processo, na } \\
\text { observação, no desenvolvimento, interação e aprofundamento e } \\
\text { ampliação de conceitos, envolvendo o desenvolvimento de projetos, } \\
\text { solução a desafios/problemas/casos, atuação nos espaços de interação } \\
\text { e nas produções disponibilizadas nos webfólios. }\end{array}$ \\
\hline $\begin{array}{l}\text { Permite aquisição de conhecimento em qualquer lugar, a qualquer } \\
\text { hora. Não é linear, nem previsível, quebra a ideia de caminhar do mais } \\
\text { fácil para o mais difícil. }\end{array}$ \\
\hline
\end{tabular}




\begin{tabular}{|c|c|}
\hline Aprendizagem autônoma & $\begin{array}{l}\text { Possibilita aos alunos encontrarem suas próprias fontes para ampliar } \\
\text { sua aprendizagem, independente de outras pessoas. Os alunos } \\
\text { contribuem com o grupo, com suas descobertas. Há espaços e } \\
\text { condições para que qualquer questão possa ser colocada e as respostas } \\
\text { possam ser construídas. }\end{array}$ \\
\hline Reflexão & $\begin{array}{l}\text { Possibilita ao professor auxiliar os estudantes no processo de } \\
\text { estabelecer relações entre o feedback de suas ações com os objetivos } \\
\text { definidos. }\end{array}$ \\
\hline $\begin{array}{l}\text { Autoria coletiva de } \\
\text { avaliações }\end{array}$ & $\begin{array}{l}\text { Permite gerenciar (definir coletivamente, inserir, consultar, alterar e } \\
\text { excluir) modalidades, instrumentos e critérios de avaliação. }\end{array}$ \\
\hline Auto avaliação & $\begin{array}{l}\text { Permite registrar e consultar relatos sobre o processo de aprendizagem } \\
\text { individual conforme critérios preestabelecidos. Permite que o } \\
\text { professor/orientador realize comentários em cada um dos critérios e } \\
\text { dê retorno ao grupo. Permite ao participante complementar a fim de } \\
\text { esclarecer as suas colocações com relação a um determinado critério } \\
\text { ou a fim de responder aos comentários do professor/orientador. }\end{array}$ \\
\hline Avaliação em grupo & $\begin{array}{l}\text { Permite registrar e consultar relatos sobre o processo de aprendizagem } \\
\text { de um grupo segundo critérios preestabelecidos. Permite que o } \\
\text { professor/orientador realize comentários em cada um dos critérios e } \\
\text { dê retorno ao grupo. Permite ao grupo complementar a fim de } \\
\text { esclarecer as suas colocações com relação a um determinado critério } \\
\text { ou a fim de responder aos comentários do professor/orientador }\end{array}$ \\
\hline $\begin{array}{l}\text { Avaliação pelo professor/ } \\
\text { orientador }\end{array}$ & $\begin{array}{l}\text { Permite registrar e consultar a avaliação do processo de aprendizagem } \\
\text { individual pelo professor/orientador segundo critérios } \\
\text { preestabelecidos. Permite que o participante realize comentários em } \\
\text { cada um dos critérios avaliados pelo professor/orientador. O } \\
\text { professor/ orientador pode complementar a fim de esclarecer as suas } \\
\text { colocações com relação a um determinado critério ou a fim de } \\
\text { responder os comentários do participante. }\end{array}$ \\
\hline Avaliação da comunidade & $\begin{array}{l}\text { Permite registrar e consultar relatos sobre o processo de aprendizagem } \\
\text { da comunidade como um todo, segundo critérios preestabelecidos. } \\
\text { Permite que o professor/orientador realize comentários em cada um } \\
\text { dos critérios e dê retorno à comunidade. Os membros da comunidade } \\
\text { podem complementar a fim de esclarecer as suas colocações com } \\
\text { relação a um determinado critério ou a fim de responder aos } \\
\text { comentários do professor/orientador }\end{array}$ \\
\hline Avaliação do sistema & $\begin{array}{l}\text { Permite registrar e consultar opiniões, sugestões e críticas sobre o } \\
\text { sistema, conforme critérios preestabelecidos. }\end{array}$ \\
\hline $\begin{array}{l}\text { Ferramenta de criação de } \\
\text { testes e provas }\end{array}$ & $\begin{array}{l}\text { Permite a criação de testes e provas com questões de diversas } \\
\text { naturezas (fechadas, abertas, múltipla escolha). Permite a criação de } \\
\text { um banco de dados de questões para geração automática de provas e } \\
\text { testes. Permite que a correção de provas e testes possa ser feita e } \\
\text { armazenada dentro do próprio ambiente do curso. Oferece a } \\
\text { possibilidade de testes nos quais as respostas possíveis podem ser } \\
\text { visualizadas pelo aluno, permitindo feedback automático (auto- } \\
\text { instrucional). }\end{array}$ \\
\hline Histórico qualitativo & $\begin{array}{l}\text { Permite consultar e acompanhar as atividades desenvolvidas por cada } \\
\text { um dos usuários do AVA. }\end{array}$ \\
\hline Histórico quantitativo & $\begin{array}{l}\text { Permite acessar dados estatísticos das atividades desenvolvidas } \\
\text { durante o curso. }\end{array}$ \\
\hline $\begin{array}{l}\text { Personalização dos } \\
\text { relatórios de avaliação }\end{array}$ & $\begin{array}{l}\text { Os relatórios de avaliação podem ser estruturados e personalizados de } \\
\text { acordo com os interesses e necessidades do professor/orientador. }\end{array}$ \\
\hline
\end{tabular}

Fonte: Adaptado de Schlemmer; Saccol; Garrido, 2007.

A partir do quadro 2 apresentado por Schlemmer, Saccol e Garrido (2015b), observa-se que os indicadores propostos para a avaliação dos AVAS, tem como propósito a melhoria da qualidade da aprendizagem, a partir do paradigma da sociedade em rede. $O$ modelo apresenta uma linguagem simples que permite às equipes interdisciplinares de 
avaliadores, composta por educadores ou pedagogos, alunos, profissionais de Tecnologia da Informação (TI) e gestores, verificarem os elementos didáticos-pedagógicos. Em outras palavras, constata-se que o cerne da avaliação está centralizado no processo de ensino e de aprendizagem.

\title{
3 METODOLOGIA
}

O método utilizado para a realização desta reflexão é o bibliográfico, no qual se fez uma revisão de literatura sobre a temática "Avaliação de AVAS" a partir do modelo interacionista/construtivista, dando-se mais ênfase aos estudos realizados pelos seguintes autores: Britain e Liber (2015), Galvão et al. (2013), Oliveira, Costa e Moreira (2001), Schlemmer (2001, 2002, 2005), Schlemmer, Saccol, Garrido (2015a, 2015b), Schlemmer e Fagundes (2000), Trein e Schlemmer (2015).

Neste contexto, a pesquisa bibliográfica à luz de Gil (2010):

\begin{abstract}
é desenvolvida a partir de material já elaborado, constituído principalmente de livros e artigos científicos [...] e a principal vantagem da pesquisa bibliográfica reside no fato de permitir ao investigador a cobertura de uma gama de fenômenos muito mais ampla do que aquela que poderia pesquisar diretamente (Gil, 2010, p. 50).
\end{abstract}

Contudo, a pesquisa bibliográfica pode ser entendida como um estudo sistemático realizado com base em material publicado em livros, periódicos e redes eletrônicas, como por exemplo, a Internet (Vergara, 2013). Neste sentido a referida pesquisa foi utilizada para fundamentar o referencial teórico e suscitar uma reflexão sobre a relevância do modelo de avaliação interacionista/construtivista de AVAS em organizações e instituições que primam pelo ensino e educação.

\section{CONSIDERAÇÕES FINAIS}

O presente trabalho analisou reflexivamente o modelo de avaliação interacionista/construtivista de AVAS, proposto inicialmente por Schlemmer e Fagundes (2000) e apresentado na íntegra por Schlemmer (2002), os quais contribuem com novas perspectivas avaliativas, identificando aspectos qualitativos, em especial, em relação à dimensão didático-pedagógica de AVAS, partindo da premissa que estes métodos de avaliação devem estar em constante atualização para acompanharem o avanço das Tecnologias da informação e comunicação (TICs).

Nesse sentido, o modelo analisado apresenta uma nova visão epistemológica que vai além de identificar as características essenciais de um AVA, ou seja, é imprescindível saber como elas estão integradas, de modo a otimizar a aprendizagem e gestão do ambiente. É necessário desenvolver a visão sobre a avaliação, permitindo conhecer sobre os princípios educacionais que norteiam o design instrucional do sistema e como os seus usuários estão utilizando os recursos do AVA para identificar efetivamente suas necessidades e ampliá-los.

Outrossim, os parâmetros utilizados como estratégia pelo modelo de avaliação interacionista/construtivista de AVAS, sob as perspectivas: técnica, comunicacionalsocial, administrativa e didático-pedagógica possibilitam que a equipe de avaliação interdisciplinar (educadores, gestores, profissionais de Tecnologia da Informação) identifique os pontos positivos e negativos de um AVA, tendo como foco o processo de aprendizagem, a partir da análise de suas múltiplas ferramentas e funcionalidades que tem por objetivo fomentar a usabilidade, a interação, a colaboração, o desenvolvimento de habilidades e competências e a formação de conceitos, a fim de possibilitar inúmeras modalidades de aprendizagem, facultando a individualidade e capacitando o aluno a administrar autonomamente seu aprendizado e seu tempo. 
Todavia, o modelo de avaliação interacionista/construtivista por ser bastante abrangente, pode ser útil para diversas instituições de ensino e organizações que apresentem a necessidade de avaliar AVAS, seja para programas de educação formal, profissionalizantes e de educação corporativa.

O modelo pode oferecer suporte para gestores e educadores no processo decisório de escolha de um software que sirva como plataforma para cursos de EAD ou de ensino presencial. Contudo, para o caso de AVAS já implantados em Instituições, o modelo de avaliação também serve para extrair os melhores resultados a partir da análise de uso do sistema, contribuindo para a otimização das ferramentas quanto à interação, à colaboração e à aprendizagem dos usuários e, também, para a expansão das propostas pedagógicas de acordo com os objetivos educacionais que se propõe o programa/curso e a instituição e/ou organização.

Contudo, pode-se dizer que o modelo interacionista/construtivista corresponde à educação tecnológica que vivenciamos em nossa atualidade, tendo em vista que permite uma comunicação mais integrada entre as equipes técnicas, pedagógicas e, principalmente, entre os alunos, tornando rico o processo de ensino e aprendizagem. Nesse contexto, os recursos oferecidos pelo modelo construtivista possibilitam um nível de interatividade muito maior que o modelo empirista dado ao efetivo conhecimento gerado pela interatividade.

\section{REFERÊNCIAS}

BRITAIN, S.; LIBER, O. A Framework for Pedagogical Evaluation of Virtual Learning Environments. Bangor: University of Wales. Disponível em:<http://www.jtap.ac.uk/reports/htm/jtap-041.html>. Acesso em: 25 set. 2015.

CHIAROTINO, Z. Psicologia e epistemologia genética de Jean Piaget. São Paulo: EPU, 1988.

COLL, César; MONEREO, Carles. Psicologia da educação virtual: aprender e ensinar com as tecnologias da informação e da comunicação. Porto Alegre: Artmed, 2010.

GALVÃO, Maria Cecília Alves et al. Análise da dimensão didático-pedagógica em ambientes virtuais de aprendizagem. Meta: avaliação. Rio de Janeiro, v.5, n. 13, p. 1228, jan./abr. 2013.

GIL, Antônio Carlos. Métodos e técnicas de pesquisa social. 6. ed. São Paulo: Atlas, 2010.

GUIMARÃES, Ângelo de Moura; DIAS, reinildes. Ambientes de aprendizagem: reengenharia da sala de aula. In: COSCARELLI, Carla Viana (Org.). Novas tecnologias, novos textos, novas formas de pensar. 3. ed. Belo Horizonte: Autêntica, 2014.

HAGUENAUER, Cristina Jasbinschek; LIMA, Luciana Guimarães Rodrigues; CORDEIRO FILHO, Francisco. Comunicação e interação em ambientes virtuais de aprendizagem. In: CONGRESSO INTERNACONAL DE EDUCAÇÃO A DISTÂNCIA, 16., 2010, Foz do Iguaçu. Anais... Foz do Iguaçu: ABED, 2010. 11p. Disponível em: <http://www.abed.org.br/congresso2010/cd/252010213152.pdf >. Acesso em: 13 jul. 2015.

KENSKI, Vani Moreira. Educação e tecnologias: o novo ritmo da informação. 8.ed. 3 reimpr. Campinas: Papirus, 2014. 
OLIVEIRA, Celina Couto de; COSTA, José Wilson da; MOREIRA, Mercia. Ambientes informatizados de aprendizagem: produção e avaliação de software educativo. Campinas: Papirus, 2001.

PETERS, Otto. A educação a distância em transição. São Leopolodo: Unisinos, 2012.

PIVA JR., Dilermando et al. EAD na prática: planejamento, métodos e ambientes de educação online. Rio de Janeiro: Elsevier, 2011.

RUHE, Valerie; ZUMBO, Bruno D. Avaliação de educação a distância e e-learning. Porto Alegre: Penso, 2013.

SCHLEMMER, Eliane. Projetos de aprendizagem baseados em problemas: uma metodologia interacionista/construtivista para formação de comunidades em ambientes virtuais de aprendizagem. Col@bora: Revista Digital da CVA-Ricesu, v.1, n.2, nov. 2001.

AVA: um ambiente virtual de convivência interacionista sistêmico para comunidades virtuais de aprendizagem na sociedade em rede. 2001. 375 f. Tese (Doutorado em Informática na Educação) - Universidade Federal do Rio Grande do Sul, Porto Alegre, 2002.

Metodologias para a educação a distância no contexto da formação de comunidades virtuais de aprendizagem. In: BARBOSA, Rommel Melgaço (Org.). Ambientes virtuais de aprendizagem. Porto Alegre: Artmed, 2005.

SCHLEMMER, Eliane; SACCOL, Amarolinda; GARRIDO, Susane. Avaliação de ambientes virtuais de aprendizagem na perspectiva da complexidade. Disponível em: $<$ http://ltc-

ead.nutes.ufrj.br/constructore/objetos/avaliacao\%20de\%20AVAs\%20na\%20perspectiva $\% 20$ da\%20complexidade.pdf $>$. Acesso em: 03 jul. 2015a.

Um modelo sistêmico de avaliação de softwares para educação a distância como apoio à gestão de ead. Revista de Gestão USP, São Paulo, v. 14, n. 1, p. 77-91, jan./mar. 2007. Disponível em: <http://www.revistas.usp.br/rege/article/view/36592/39313>. Acesso em 03 jul. 2015 b.

SCHLEMMER, Eliane; FAGUNDES, Léa da Cruz. Uma proposta para a avaliação de ambientes virtuais de aprendizagem na sociedade em rede. Informática na educação: teoria e prática, Porto Alegre, v.3, n.1, p. 1-15, set. 2000.

TORI, Romero. Educação sem distância: as tecnologias interativas na redução de distâncias em ensino e aprendizagem. São Paulo: Editora Senac São Paulo, 2010.

TREIN, Daiana; SCHLEMMER, Eliane. Projetos de aprendizagem baseados em problema no contexto da web 2.0: possibilidades para a prática pedagógica. Revista ECurriculum, São Paulo, v.4, n.2, jun. 2009. Disponível em: <http://revistas.pucsp.br/index.php/curriculum/article/viewFile/3225/2147>. Acesso em: 27 set. 2015.

VERGARA, Sylvia Constant. Projetos e relatórios de pesquisa em Administração. 2. ed. São Paulo: Atlas, 1998. 